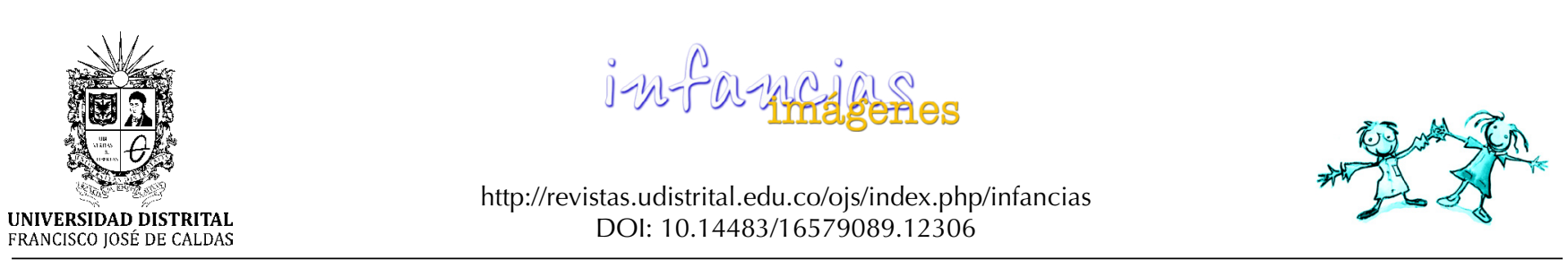

TEXTOS Y CONTEXTOS

\title{
Periferia urbana de Usme: una mirada desde la infancia escolarizada de la UPZ Comuneros*
}

\author{
Urban Periphery of Usme: A Look upon Childhood Schooling of UPZ Comuneros
}

Giovanny Eduardo Bogoya Caviedes'

Para citar este artículo: Bogoya, G. E. (2018). Periferia urbana de Usme: una mirada desde la infancia escolarizada de la UPZ Comuneros. Infancias Imágenes, 17(1), 78-89.

\section{Resumen}

El presente artículo de reflexión expone los hallazgos de una investigación desarrollada durante el año 2016 con estudiantes de básica primaria en la IED Bogotá (Colombia). Esta vivencia representó una apuesta teórica y metodológica por problematizar las tensiones que se generan entre sujeto y territorio, desde la lectura de las expresiones y los lazos sociales que construye la infancia en la periferia urbana. Para ello, se realizó una investigación con enfoque cualitativo de tipo etnográfico, apoyada en las técnicas de observación, grupos de discusión y cartografía social que dejaron como principal hallazgo el juego como el vínculo fundamental que establecen los estudiantes con el territorio.

Palabras clave: infancia; estudiantes; expresiones; periferia urbana; territorio.

Recibido: 15-julio-2017 / Aprobado: 04-febrero-2017

\begin{abstract}
This article of reflection exposes the findings of an investigation developed during the year 2016 with elementary students in the IED Diego Montaña Cuellar in the locality of Usme, Bogotá (Colombia). This experience presented a theoretical and methodological commitment to problematize the tensions generated between subject and territory, from reading of expressions and social bonds which childhood builds in the urban periphery. For this purpose, a qualitative ethnographic research was carried out, supported by observation techniques, discussion groups and social cartography which left as a main finding, that using games established a fundamental link by students with territory.
\end{abstract}

Keywords: childhood; students; expressions; urban periphery; territory.

\footnotetext{
* Este artículo es derivado del trabajo de grado titulado "Espacios rururbanos en Usme: una mirada desde los jóvenes escolarizados de la UPZ 58-Comuneros", investigación desarrollada entre los años 2015 y 2016 en la localidad de Usme, Bogotá, y presentada como requisito para obtener el título de magíster en Educación.

1 Magíster en Educación, especialista en Derechos Humanos, licenciado en Ciencias Sociales. Docente vinculado a la Secretaría de Educación del Distrito. Correo electrónico: eduardo.bogoya@gmail.com
} 


\section{Introducción}

Este documento tiene su origen en una investigación realizada durante el año 2016 con estudiantes de ciclo II $\left(3^{\circ}\right.$ y $\left.4^{\circ}\right)$ de educación básica primaria en la institución educativa distrital (IED) Diego Montaña Cuellar, de la localidad de Usme. Experiencia que centró su análisis en la lectura de las relaciones que se tejen entre infancia y territorio desde la periferia urbana de la localidad.

Ubicada en el suroriente de la ciudad de Bogotá, la localidad de Usme cumple en sus condiciones geo-espaciales con una serie de características en las que convergen dinámicas sociales propias de los bordes de la ciudad. Contexto en el cual se hace difícil determinar la frontera en la que inicia lo urbano y termina lo rural, en este caso, en la Unidad de Planeamiento Zonal 58 Comuneros (UPZ), en la que se emplaza la institución².

En medio del ambiente rural y las prácticas urbanas que surgen de la interacción en esos espacios, tienen lugar una serie de acciones que se desarroIlan en la periferia urbana de la localidad. Es aquí donde los saberes que circulan en la zona porosa de Usme y la institución educativa demandan ser analizados. Sobre este escenario surgió la siguiente pregunta problema: ¿qué tipo de lazos sociales construyen los estudiantes de básica primara del colegio Diego Montaña Cuellar en la periferia urbana de Usme?

Con la pregunta se buscó dar respuesta al objetivo general, el cual consistía en determinar de qué manera la infancia escolarizada de Usme construye lazos sociales en el escenario periférico de la UPZ 58 Comuneros. Agregado a los objetivos específicos de explorar lo periurbano, reconocer las expresiones e interpretar los vínculos que tienen lugar en el entramado infancia-territorio. Para ello, se diseñó una metodología de investigación cualitativa

\footnotetext{
2 Las unidades de planeamiento zonal (UPZ) son una subdivisión territorial de Bogotá conformada por varios barrios de una misma localidad que comparten características comunes. La finalidad de las UPZ es definir la planeación urbana que demanda la ciudad. Usme se divide en siete UPZ, entre estas la UPZ 58 Comuneros, escenario que sirvió de referente empírico para esta investigación, al ser el lugar donde se emplaza la IED Diego Montaña Cuellar. La principal norma que rige a las UPZ es el Plan de Ordenamiento Territorial (POT), por medio del artículo 49 del Decreto 190 de 2004 de la Alcaldía Mayor de Bogotá D.C.
}

de tipo etnográfico; las técnicas utilizadas fueron: observación no participante, grupos de discusión y cartografía social.

El documento se estructura en cinco apartados: primero, se contextualiza el concepto de periferia urbana (periurbanidad o periurbano) desde la territorialidad de Usme, buscando una articulación con la educación; segundo, se da lugar a la noción de infancia contemporánea y sus vínculos con el referente empírico; tercero, se presenta la apuesta metodológica implementada; cuarto, se exponen las técnicas utilizadas con sus respectivos resultados y análisis; y por último, se enseñan los hallazgos y recomendaciones que suscitaron la investigación.

\section{Periferia urbana: una aproximación conceptual}

En una ciudad con las características demográficas de Bogotá, los asentamientos sub-normales son la constante en las zonas periféricas de la capital, situación que afecta su crecimiento poblacional de una manera vertiginosa. Según datos del Dane (2011), la proyección de la población de Bogotá sin su área metropolitana para el año 2017 es de 8'080.734 habitantes. De este número, 3'323.866 residen en las zonas periféricas de la capital, cifra que representa el $41.13 \%$ del total de la población ${ }^{3}$.

Socialmente, ha sido difícil asimilar a la periferia como parte de la ciudad y por el contrario suele excluirse de la misma, al entenderse como una zona aislada a los intereses de los citadinos. Además de su difícil aceptación social, teóricamente es difícil categorizarla y suele asociarse con conceptos comúnmente usados en los estudios urbanos territoriales; términos como: suburbano, conurbano, expansión urbana, área metropolitana e interface

\footnotetext{
Aunque no hay una definición general sobre cuáles localidades de la ciudad son consideradas periféricas, sí existen categorías que permiten clasificar algunas de estas como parte de la periferia, las cuales son: distancia desde el centro de la ciudad, pocas vías de acceso, inaccesibilidad del transporte masivo, carencia de servicios públicos, hibridación entre lo urbano y lo rural, escasos escenarios para la recreación, cultura o entretenimiento, ausencia de centros de salud, urbanizaciones informales, asentamientos masivos de población desplazada, mal uso del suelo, condiciones de vulnerabilidad a causa de desastres naturales. Desde estas condiciones, las localidades en las que más se presentan estas características son: San Cristóbal, Usme, Bosa, Suba, Ciudad Bolívar, Sumapaz. Véase Secretaría Distrital de Planeación (2010).
} 
ciudad-campo tienen validez al explicar las relaciones que se entretejen en las zonas contiguas de la ciudad, pero que legalmente hacen parte de esta.

Aunque la periferia se incrusta en las ciudades y limita con la ruralidad, sus dinámicas no son características del centro de la ciudad y tampoco de la estructura del agro. Es un escenario que suele definirse con notables contrastes de desigualdad social:

Los perímetros urbanos de sus ciudades son superados por asentamientos que enfrentan todo tipo de limitantes, artificiales y naturales, con sus precarias construcciones, incluso en zonas de alto riesgo o con favorecidos sectores de exclusivos grupos sociales, que evidencian el alto fraccionamiento de las sociedades suramericanas. (Bahamón, 2009, p. 48)

Lo anterior evidencia las características generalizadas que otorgan los análisis sociales sobre estas zonas periféricas.

La formación de un espacio periurbano debe ser contextualizado desde tres bloques temáticos (espacial, económico y social) que tienen su génesis en las zonas próximas a las ciudades: "Lo periurbano es un espacio genérico que rodea a cualquier ciudad independientemente de su actividad, función o tipo de ocupación" (Delgado, 2003, p. 28). Según esto, cualquier lugar alrededor de la ciudad es periurbano sin importar si este responda a un poblamiento rural, urbano-rural, o propiamente urbano. Siguiendo la misma definición, todas las ciudades deben tener lugares periféricos que alimentan las cotidianidades de su centro y aunque las zonas limítrofes a la urbe visualmente puedan ser diferentes según el contexto, todas cumplen las mismas funciones, debido a que son el lugar de habitad de trabajadores, estudiantes y personas que se dirigen al centro de la ciudad para realizar sus labores o diligencias.

Pero, ¿de qué manera se da el proceso de expansión de la ciudad hacia los territorios adyacentes, ahora llamados periurbanos? ¿Sobre qué lógicas se urbaniza más allá del centro de las ciudades? ¿Cómo definir la aparición de los lugares periurbanos? Según Aguilar (2002), la expansión de la ciudad es un proceso histórico, continuo y sistemático que ha caracterizado las condiciones de poblamiento de las ciudades latinoamericanas y de los países en vía de desarrollo. Así lo expone el geógrafo mexicano:

En términos territoriales, de un espacio metropolitano relativamente compacto, la ciudad contemporánea presenta una expansión más policéntrica, creando un patrón más asociado con redes y con límites y fronteras menos precisas difícilmente definibles. Lo anterior genera un patrón de expansión con tendencias de dispersión urbana que incorpora progresivamente pequeños pueblos y periferias rurales dentro de un sistema metropolitano cada vez más amplio y complejo. (Aguilar, 2002, p. 123)

Desde esta perspectiva, las urbes contemporáneas tienden a expandirse hacia los lugares colindantes acaparando zonas consideradas rurales, criticas, marginadas o de difícil acceso, las cuales se incorporan con el pasar del tiempo a ciertas prácticas de la ciudad y responden a sus necesidades. Es así como surge la periferia que ahora hace parte trascendente de la capital, sin periferia no hay ciudad y sin ciudad no hay periferia.

Siguiendo con la explicación sobre lo periurbano, se hace necesario resaltar que no toda periferia es considerada marginal; por el contrario, hay lugares ubicados en los extremos de las ciudades que cumplen ciertas características que las determinan como lugares progresivos. En estos se desarrollan procesos de gentrificación, dando como resultado espacios propicios para la residencia o esparcimiento de una clase social acomodada ${ }^{4}$. Lo anterior responde a grupos privados que intervienen en las zonas como una estrategia de crecimiento e inversión de su capital económico,

La inversión extranjera ha tenido importantes impactos en la periferia metropolitana; estos impactos

\footnotetext{
4 La gentrificación hace referencia a un proceso de transformación urbana en el que la población original de un sector es desplazada, ya sea por compra, embargo, expropiación o expulsión, por otra de un mayor nivel adquisitivo; , esto trae como consecuencia la renovación arquitectónica de dicho sector debido a la inversión privada. Aunque los primeros procesos de gentrificación se ubican en la Inglaterra industrial del siglo XIX, el concepto es estudiado a partir de la segunda mitad del siglo XX por la socióloga británica Ruth Glass (1964) y teorizado décadas posteriores por el también británico Michael Pacione (2001).
} 
incluyen sobre todo desarrollos residenciales privados, generalmente con sitios recreativos como campos de golf o country clubs; grandes obras de infraestructura, por ejemplo, aeropuertos y complejos corporativos de oficinas o centros comerciales en las localizaciones más accesibles. (Aguilar, 2002, p. 126)

A partir de estas lógicas de apropiación y poblamiento de los bordes urbanos, fue necesario cuestionarse sobre las tensiones que se generan entre los sujetos y el territorio. Condiciones conjugadas que llevaron a indagar sobre las expresiones que desarrolla determinada parte de la población de la localidad asentada en la UPZ, en específico la comunidad escolar del colegio Diego Montaña Cuellar. Tomando el campo de lo pedagógico, ¿qué vínculos tendría lo periurbano con la educación? Al asumir la educación como un proceso por el cual la sociedad forma a sus sujetos, y que tal proceso está mediado por el territorio en cuanto que la misma sociedad se organiza geo-culturalmente, lo periurbano establece una estrecha relación con la educación.

En consecuencia, las dinámicas en la periferia urbana devienen en prácticas culturales que configuran subjetividades, es decir, que derivan en procesos educativos, aunque no obligatoriamente de carácter institucional, sí de enculturación social. Pero, esta conexión entre lo periurbano y educación puede ser más evidente en la institucionalidad escolar si los colegios presentes en la periferia urbana extienden proyectos pedagógicos territorializados, lo que conllevaría a consecuencias directas en el currículo. Ejemplo de ello, el proyecto ambiental (Prae) de una institución escolar urbana o rural tendrá diferencia con uno de la periferia urbana, debido a que este responderá a contextos geográficos, culturales, ambientales, económicos, laborales y de poblamiento específicos.

La escuela como constructo social se encuentra permeada por los diversos tejidos culturales en los cuales actúan sus participantes. El territorio no es indiferente a estas circunstancias, las prácticas que circulan en la periferia urbana difieren de otros saberes que transitan en un espacio completamente rural o totalmente urbano. Elementos como: las estrategias didácticas, el plan de estudios, el proyecto educativo institucional, el horizonte institucional, los proyectos transversales, el sistema de evaluación, entre otros, deben ser consecuentes a las características del contexto y responder a sus posibles necesidades.

La infancia contemporánea y sus vínculos con los bordes urbanos de Usme En relación con la población protagonista de este estudio, la infancia actualmente es asumida como un sujeto autónomo en el marco de una esfera social. Se constituye en un proceso en el que intervienen: sujetos, instituciones y saberes, que al unísono conjugan una serie de discursos y prácticas que al trenzarse conforman la infancia contemporánea (Zuluaga, 1999). Desde esta mirada, existiría una tensión entre la infancia y la periurbanidad, la cual puede ser complejizada para entender dicho proceso desde el territorio, en este caso, desde la zona porosa de Usme; ambiente en que se dinamizan discursos y prácticas que al interior de la escuela configuran saberes que, a su vez, son expresados por la población estudiantil, como es el caso, de la niñez escolarizada de la UPZ.

En la contemporaneidad, la infancia se encuentra inmersa en un proceso de socialización, naturalización y subjetivación que da lugar a un nuevo tipo de sujeto. Tomando a Jiménez (2007):

La infancia actual es aquella que lucha por conquistar su identidad en un contexto urbano mucho más individualizado, en el que la familia nuclear se encuentra amenazada; las crisis entre las generaciones cada día son más estrechas, y los niños y los jóvenes, aunque cada día más dependientes en el orden social de su estructura familiar, son a la vez más precoces. (p. 153)

Desde este panorama, el factor de socialización de la infancia se fragmenta y brinda pocos espacios de entretenimiento en colectivo.

Es aquí que el territorio entra a desequilibrar estas dinámicas, debido a que las condiciones geo-culturales de Usme brindan espacios de socialización enmarcados por la autonomía del sujeto: 
La infancia contemporánea, vista desde la perspectiva de la ciudad, ha vivido un particular proceso de socialización, en el cual la calle cumple un papel fundamental como espacio de socialización en el que se viven aventuras, se entretejen relaciones, se consiguen amigos y se conquista buena parte de la identidad, el yo social. (Jiménez, 2007, p. 172)

Por ello, en la periferia urbana de la localidad se generan una suerte de procesos colectivos en los cuales los niños generan identidad frente al territorio. Según Jiménez (2008), asumir la infancia como sujeto implica comprender un proceso de naturalización, socialización y subjetivación, en el cual participan sujetos, instituciones y saberes que en un cruce de fuerzas consolidan la infancia contemporánea. Estrategias alternas de socialización que dan lugar a un yo social, en este caso, enmarcado en la espacialidad de Usme.

Para ampliar esto último, en la periferia urbana de la localidad se generan expresiones de pertenencia y uso del territorio que se pueden observar en la infancia: "El territorio genera una profunda valoración emocional, su historia, las tradiciones, paisajes, modos de habitar el espacio y todas aqueIlas manifestaciones culturales dan singularidad a una región o localidad" (Molina, 2011, p. 9). Valoración que se materializa en las conductas desde y hacia el territorio que exteriorizan los estudiantes. Surgen entonces juegos, maneras de hablar, formas de vestir, corporalidad, usos de los espacios, valores o antivalores respecto al ambiente, actividades productivas, agrado o desagrado por vivir allí que identifican a los sujetos que en él conviven, específicamente en la infancia presente en la UPZ.

Entonces, se puede pensar que dichas expresiones sobre la periferia derivan de la transformación ocurrida en los últimos años en Usme, la cual conIleva a cambios en las rutinas culturales, sociales y económicas de la localidad que directa o indirectamente permean las subjetividades de la infancia, en concreto, en aquella que hace presencia al interior del colegio. De la misma manera, en el marco de un entramado geo-cultural y territorial, estas expresiones se asocian a la educación como proceso social, debido a que se originan por medio de estrategias de enculturación y socialización constantes que provienen de procesos de subjetivación que se construyen con los otros, el yo social.

En general, la infancia contemporánea demanda nuevos espacios de enculturación en el escenario de lo público, dirigiendo su mirada hacia la consolidación de sujetos constructores de nuevas realidades y subjetividades:

La Infancia contemporánea al demandar su presencia en el escenario público presiona otras miradas que sobre ella se tiene aún desde el desconocimiento en su dimensión, no sólo, de escolar uniformado y de hijo, sino en su dimensión de niño como constructor de una nueva realidad y de una nueva subjetividad. (Infante y Jiménez, 2006, p. 279)

Subjetividades que en la zona periférico de Usme cobran protagonismo al establecerse diversas formas de conexión sobre el territorio, vínculos que giran en torno a unas narrativas socioculturales de la infancia con su entorno.

En conclusión, existe un nudo entre la periferia y la infancia, en la medida que esta última tiene como una de sus plataformas de acción los bordes urbanos de Usme en donde hacen presencia: sujetos, saberes e instituciones que al conjugarse establecen discursos y prácticas mediados por las condiciones geo-culturales de la UPZ Comuneros. En esta la infancia genera narrativas que a su vez suscitan tensiones que trascienden los muros escolares. Por lo anterior, fue provechoso indagar sobre las condiciones en que se entreteje este lazo social, desde la lectura del territorio por parte de los estudiantes.

\section{Metodología}

Con los propósitos de explorar el territorio, reconocer las expresiones e interpretar los lazos que tejen los estudiantes dentro de la UPZ Comuneros, se implementó una metodología de investigación cualitativa de tipo etnográfica:

En un sentido mínimo, la etnografía puede entenderse como un proceso de 'documentar lo no-documentado'. La base de este proceso es el trabajo de campo y la subsecuente elaboración de los registros y del diario de campo [...] Aunque también es 
importante usar otros procedimientos en el campo (mapas, entrevistas, documentos, redes, juegos) que son más sistemáticos. (Rockwell, 2009, p. 48)

Más allá de pertenecer a una cultura sobre las zonas limítrofes de la ciudad, la infancia re-significa discursos y prácticas que producen conocimiento en colectivo, lo que da lugar a la apropiación de saberes que encuentran en el territorio una plataforma de acción y estrategias de empoderamiento.

Desde esta mirada, apoyada en las subjetividades y en aras de rescatar lo humano, he tomado los alcances de la etnografía crítica en el campo de la educación, advirtiendo que toda investigación etnográfica en lo educativo no siempre conduce a una etnografía crítica. Por lo anterior, comparto con Suárez:

Es necesario que dirijamos la mirada al actor y no tanto al resultado; obviamente no podemos pretender que todas las investigaciones logren el mismo grado de involucramiento, unas quedarán más en el nivel descriptivo y otras buscan una interpretación más profunda del fenómeno. (2012, p. 22)

Entonces, la propuesta giró en torno a las subjetividades de los protagonistas y se buscó complejizar los procesos sociales que desarrollan los estudiantes dentro de la espacialidad de Usme.

Es aquí que el ejercicio empírico cobra notoriedad al valerse de procedimientos para documentar las trayectorias de los protagonistas. Por ello, a partir del trabajo de campo se buscó reconocer las experiencias y narrativas que presentan los estudiantes dentro del territorio, retomando a Rockwell:

Lo que de hecho se hace en el campo depende de la interacción que se busca y se logra con personas de la localidad y de lo que ellos nos quieran decir y mostrar. Intervienen nuestros propios procesos inconscientes, las formas en que manejamos nuestras angustias en el trabajo y las interpretaciones de la situación que apenas articulamos como tales. Influyen las posturas políticas y los compromisos éticos que asumimos. (2009, p. 49)

En esta investigación, los estudiantes hacen parte de un grupo social inmerso en una cultura, en la cual tienen lugar procesos sociales que afectan los comportamientos, costumbres o hábitos de los integrantes del grupo. Es allí que la observación e interacción con la comunidad permiten nuevas formas de entendimiento en torno a las relaciones que establecen los sujetos con el territorio. Conexiones que el etnógrafo crítico vive dentro de un proceso dialéctico, reflexivo y de constante cuestionamiento (Vargas, 2016).

Concretamente en el campo de lo educativo, la etnografía permite la interpretación de fenómenos sociales a través de la observación del contexto y de las narrativas de los participantes, persiguiendo un proceso de construcción teórica paralelo a la investigación empírica (Rockwell, 1993). Es así como a partir de la observación y el análisis en la etnografía se va hilando unas categorías teóricas que permiten el acercamiento al fenómeno social, variables que a partir del territorio cobran protagonismo en los procesos sociales de la infancia.

Continuando con lo educativo, se asumió la investigación cualitativa como "Una actividad sistemática orientada a la comprensión en profundidad de fenómenos educativos y sociales, a la transformación de prácticas y escenarios socioeducativos, a la toma de decisiones y también hacia el descubrimiento y desarrollo de un cuerpo organizado de conocimiento" (Sandín y Paz, 2003, p. 123). Con su aplicación se buscó reflexionar sobre los procesos sociales y relaciones de poder que entrelazan los estudiantes en el territorio. En consecuencia, con esta metodología se establecieron estrategias para acercarse a la complejidad de lo periurbano desde una parte de la población infantil escolarizada de la localidad.

La investigación etnográfica implica la observación de un fenómeno social desde la lectura de categorías teóricas, las cuales pueden aparecer a lo largo del proceso, aunque no siempre deben estar atadas a conductas observables. De allí la importancia en la espontaneidad de los sujetos, de esa condición humana que no se sesga a una tabula rasa. De este modo, se profundiza en las cualidades que no son observables a simple vista y que los sujetos exteriorizan como formas particulares dentro de un proceso social, en este caso, la infancia escolarizada dentro de la UPZ Comuneros. 
En síntesis, con la etnografía se apuesta por la apertura hacía otros caminos de investigación, trayectos que están en constante movimiento y que a partir del trabajo empírico buscan establecer nuevas relaciones con el conocimiento. Como lo indica Rockwell (2009):

Considero que se ha hecho análisis etnográfico solo cuando se modifica sustancialmente la concepción inicial del proceso que se estudia; cuando, a consecuencia de la construcción de nuevas relaciones, se puede dar mejor cuenta del orden particular, local y complejo del proceso estudiado; cuando la descripción final es más rica y más coherente que la descripción inicial; cuando se abren nuevos caminos de investigación, siempre en proceso de construcción, siempre inconclusos. (p. 67)

Por todo lo anterior, los desafíos de la investigación fueron explorar, reconocer e interpretar los lazos sociales que hilvana la infancia, inmersa en un proceso social sobre la zona porosa de Usme. Las técnicas e instrumentos de recolección de información propuestas fueron: observación no participante (matriz de observación), grupos de discusión (matriz textual) y cartografía social (mapas).

Respecto al trabajo de campo, el principal escenario que sirvió como referente empírico fue la sede A de la IED Diego Montaña Cuellar, ubicada en el barrio Usminia, dentro de la UPZ Comuneros. La intervención con la población se facilitó debido a que laboré en la institución desde el año 2013 como docente de básica primaria en las áreas de Ciencias Sociales y Humanidades, compartiendo a diario con los estudiantes; situación que me permitió hacer un ejercicio más cercano con la comunidad estudiantil.

Además de la institución, se acudió a escenarios alternos dentro de la UPZ, siendo estos: zonas verdes en los parques barriales Usminia, Villa Anita y Villa Alemana, el parque ambiental Cantarrana y Usme centro, a los cuales se accedió en compañía de los estudiantes y otros docentes del área de Ciencias Sociales. Lo anterior, en el marco de una estrategia pedagógica de reconocimiento del territorio.

\section{Leyendo la periurbanidad, un ejercicio} desde la infancia

Cerca de mi casa hay muchas fincas, se ven vacas y animales como en el campo, se respira aire puro y es muy tranquilo. Pero más abajo hay mucha inseguridad, roban seguido, cuando llueve huele muy a feo, es muy peligroso, como si estuviera en la ciudad.

(Valery Andrea Barraza García)

Observación

Se llevó a cabo en el año 2016 (periodos lectivos) en el parque infantil dentro de la institución educativa, el parque ambiental Cantarrana (dos visitas) ubicado en la UPZ y Usme pueblo. Escenarios que se encuentran rodeados de amplias zonas verdes, el rio Tunjuelo, unidades residenciales y vías vehiculares.

Los protagonistas fueron los estudiantes de los grados $3^{\circ}$ y $4^{\circ}$ de la jornada tarde de la institución, cuyas edades oscilan entre los 8 a 10 años, algunos viven en zonas rurales y otros en sectores urbanos de Usme; todos habitantes de la UPZ. La observación dentro del colegio se realizó en el descanso, allí las niñas y los niños se mostraron alegres jugando en el parque y compartiendo con sus amigos. Las visitas — tanto al Cantarrana como a Usme centro- se realizaron tres viernes entre la 1 y 5 de la tarde. En el Parque Cantarrana los estudiantes jugaron en las canchas, potreros y árboles, sus expresiones fueron de alegría e integración; la llegada al lugar se hizo a pie desde el colegio. Respecto a la visita a Usme centro, se llegó en un bus contratado por la institución, se realizó una visita guiada a cargo de los docentes en la que se recorrió la plaza fundacional y las calles céntricas del pueblo. Durante el trayecto los estudiantes se mostraron curiosos e interesados por conocer más sobre Usme.

La estrategia utilizada en los tres escenarios consistió en observar a los estudiantes cuando compartían entre ellos. Con esta técnica se buscó reconocer las expresiones que asumen los estudiantes dentro del territorio de la UPZ. Lo visto se sistematizó en la matriz de observación; después de su desarrollo se pudo concluir que:

A. Las niñas y los niños hacen uso de los espacios naturales para jugar y compartir entre amigos; algunos proponen juegos de contacto físico 
como: cogidas, congelados, policías y ladrones, fútbol, ponchados y saltar lazo. Mientras un pequeño grupo descansaba o jugaba con tarjetas tipo cartas. Con ello, se ve una apropiación del territorio en la mayoría de los estudiantes al aprovechar los espacios verdes para su esparcimiento.

B. La presencia de elementos propios del campo como fincas, agricultura, campesinos y animales son aceptados por la mayoría de la niñez y no generan ninguna novedad. Los entienden como parte intrínseca de la comunidad debido a que cierto número de niñas y niños tienen vivencias cercanas al campo, ya que residen o tienen familiares que trabajan en fincas del sector.

C. Se observan expresiones hacía el cuidado de la naturaleza que se manifiestan en la separación adecuada de los residuos sólidos. Después de compartir el refrigerio, los estudiantes depositaron los desechos en las canecas correspondientes, esto se observó en las visitas al parque Cantarrana.

D. Los protagonistas se apropian de un lenguaje citadino característico de la zona que habitan. Aparecen expresiones que representan amistad o compañerismo, como: amigo, chinche o sumercé; algunas expresan desagrado, como: pescado (feo), curtir (fastidiar); y otras representan acciones o actividades: roli (vuelta), güiro (pelea), tapador (arquero de fútbol). Las anteriores se utilizan para relacionarse entre pares, esto se observa en la instancia dentro del colegio.

E. A gran parte de los estudiantes les agrada acudir a la institución, siendo el lugar donde más comparten con sus compañeros. Asimismo, las salidas pedagógicas (parque Cantarrana y Usme pueblo) generan agrado en la comunidad estudiantil. A cuatro niñas no les agradó la salida a Usme centro; expresaron su cansancio por la caminata y se observaron desmotivadas durante el recorrido.

F. Los lugares que más disfrutan los estudiantes son las zonas verdes, siendo estos en los que interactúan libremente. Mientras unos grupos jugaban, otros descansaban en los pastales; esto se reflejó dentro de la institución y en las visitas al parque Cantarrana.
En la figura 1 se observan algunos estudiantes compartiendo en uno de los espacios que ofrece el colegio. Las niñas y los niños se divierten con un balón, apropiándose de la territorialidad del colegio, siendo el juego la principal conexión que se establece entre la infancia y el territorio dentro de la institución. Al fondo se divisa parte de la zona periférica de la UPZ; en ella hay viviendas del barrio Usminia, explotación del suelo para la construcción de apartamentos, fincas, presencia de animales propios del campo, extensas zonas verdes, montañas, cultivos y naturaleza rural que tiene su espacio dentro de lo urbano, es decir, lo periurbano dentro de Usme.

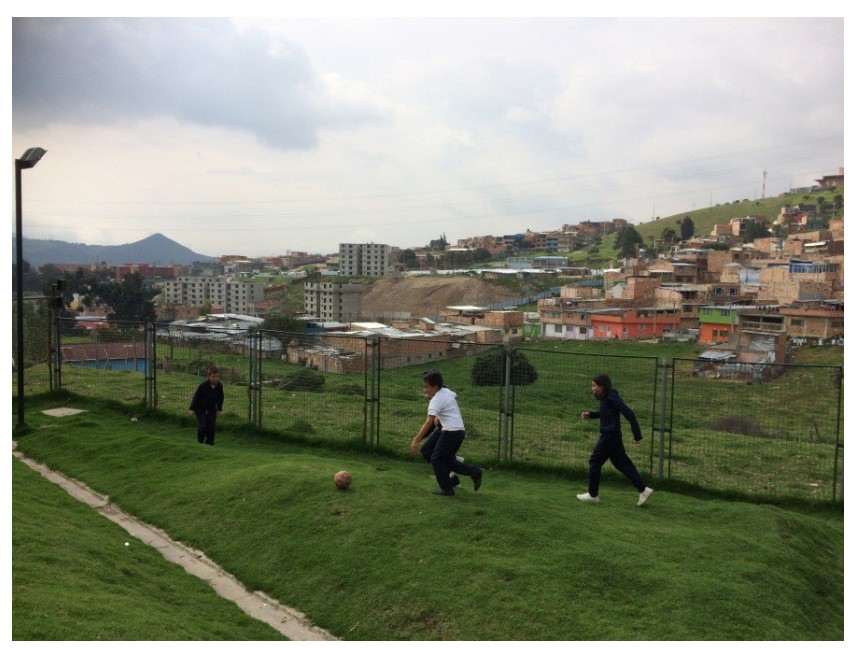

Figura 1. Observación.

Fuente: archivo personal del autor (2016).

Grupos de discusión

En la biblioteca principal de la sede A del colegio se organizaron cuatro grupos de 10 estudiantes cada uno, los cuales cursaban grado $4^{\circ}$ (401 y 402) en la jornada tarde y a quienes acompañe como profesor en el área de Ciencias Sociales. Los criterios de selección de los participantes fueron: estudiantes entre 8 y 10 años de edad que residen en barrios pertenecientes a la UPZ, cuya trayectoria escolar responde a un proceso de formación iniciado en la institución desde el ciclo I y han sido receptivos en actividades de integración propuestas por la institución, provenientes de familias monoparentales y clasificadas en estratos socioeconómicos 1 y 2. 
Se realizó una entrevista grupal por separado durante 60 minutos, iniciando con la siguiente pregunta orientadora: ¿qué tipos de lugares observa cerca al colegio?, descríbalos. Seguido de ello, se socializaron otras preguntas que buscaron ofrecer variables de respuestas a los objetivos específicos de la investigación, algunas de estas fueron: ¿considera que usted vive en el campo, en la ciudad, o en medio de los dos?, explique su respuesta; en sus tiempos libres, ¿qué tipo de actividades realiza cerca al lugar donde vive?; cerca de su casa o colegio, ¿qué tipo de animales existen?; en su casa, ¿usted o su familia hacen actividades propias del campo?, de ser afirmativo, descríbalas, le gusta hacerlo; por medio de un ejemplo, mencione ¿qué lugares considera usted urbanos y cuáles considera rurales?; cuando está en el parque con sus amigos ¿a qué juegan?

En un segundo encuentro se socializaron las respuestas dadas por los participantes, dando paso a una discusión colectiva en la que los estudiantes se mostraron participativos, exponiendo sus diferencias en torno a las características físicas de territorio, en especial, de aquellos lugares que entendían como rurales (fincas, potreros, montañas) frente a otros que asumían como urbanos (barrios, tiendas, colegios). Por último, se analizó la información recopilada en la matriz textual. Esta técnica dejó los siguientes resultados de los estudiantes:

A. Expresan que viven en medio del campo y la ciudad; reconocen que cerca al colegio se desarrollan dinámicas propias de los dos ambientes. Situación que se vislumbra al expresar que en el sector existen: fincas, ríos, quebradas, animales de campo, amplias zonas verdes $y$ montañas, pero también hay barrios, parques, colegios, contaminación y vehículos.

B. Comprenden que Usme hace parte de Bogotá, pero expresan que es un lugar muy alejado del centro de la ciudad. Por lo general, asocian esta idea con la condición laboral de sus familias y los tiempos de desplazamiento que estas utilizan desde sus lugares de trabajo hasta sus casas.

C. Identifican animales propios del campo como: vacas, burros, caballos, ovejas, marranos, y gallinas, principalmente.
D. Registran la existencia de fincas y campesinos en el sector, describiendo sus principales características. Muchos de los estudiantes conviven con familiares que trabajan en la agricultura existente dentro de la UPZ.

E. Indican que en la zona hacen presencia gran cantidad de niñas y niños, pocos escenarios culturales como bibliotecas, ludotecas o teatros, no existen centros comerciales, hay un mal servicio de transporte y vías vehiculares, deficiente prestación de servicios básicos como agua y electricidad, contaminación ambiental debido a la cercanía del relleno Doña Juana, inseguridad, venta de drogas, invasión de terrenos para la construcción de viviendas; condiciones comunes en la periferia urbana.

\section{Cartografía social}

Su implementación facilitó la lectura del territorio fronterizo a la institución desde la mirada de los estudiantes. La técnica se estructuró en tres fases: primero, selección e inducción de los participantes; segundo, recorrido por el territorio; y tercero, elaboración de mapas. Se construyeron cuatro mapas (reconociendo el sector urbano, dibujando la ruralidad, representando la periferia urbana, lugares de mayor y menor gusto) en donde los estudiantes colectivamente simbolizaron los espacios más relevantes del sector. En esta técnica participaron 20 estudiantes del grado $4^{\circ}$ (401 y 402) de la jornada tarde de la institución, los cuales tenían un buen desempeño académico y eran propositivos en el área de Ciencias Sociales. El recorrido se realizó en un lapso de cuatro horas entre la 1 y 5 de la tarde, la ruta escogida fueron los barrios: Usminia, Villa Anita y Antonio José de Sucre; aquí los participantes expresaron familiaridad con el territorio, pues la mayoría habitan en este sector.

Para la última fase se organizaron cuatro equipos de trabajo cada uno integrado por cinco estudiantes, se les brindó los materiales y cada grupo asumió una función, empoderándose del ejercicio de mapeo: hacían preguntas y discutían sobre aquellos lugares que debían representar en los mapas. En el desarrollo de las tres fases los integrantes estuvieron dispuestos a la ejecución de las actividades propuestas, se mostraron activos, receptivos, 
participativos y colaborativos. El uso de la cartografía arrojó las siguientes conclusiones:

A. Los espacios más frecuentes que se representaron en los mapas fueron: el colegio, zonas verdes, parques (barriales y Cantarrana), urbanizaciones, fincas y el río Tunjuelo.

B. Los lugares periurbanos ubicados en los mapas corresponden al paisaje entre la institución educativa y los barrios en los que viven los estudiantes. Siendo estos: el colegio Diego Montaña Cuellar, rio Tunjuelo, quebrada Chuniza, parque ambiental Cantarrana, parque Villa Alemana, parque de Usminia y la carrera 2 Este.

C. Las figuras más visibles en los mapas (véase figura 2) son: árboles, montañas, fuentes hídricas, animales (vacas, caballos, burros, ovejas, marranos, conejos y gallinas), casas, fincas, zonas verdes y vehículos.

D. Los estudiantes representaron un equilibrio entre el paisaje natural y construido, como se puede observar en la figura 2, en la cual se graficó en medida similar lo rural: bosques, fincas, agricultura, animales, ríos y montañas; y lo urbano: avenidas, buses, urbanizaciones, parques y el colegio.

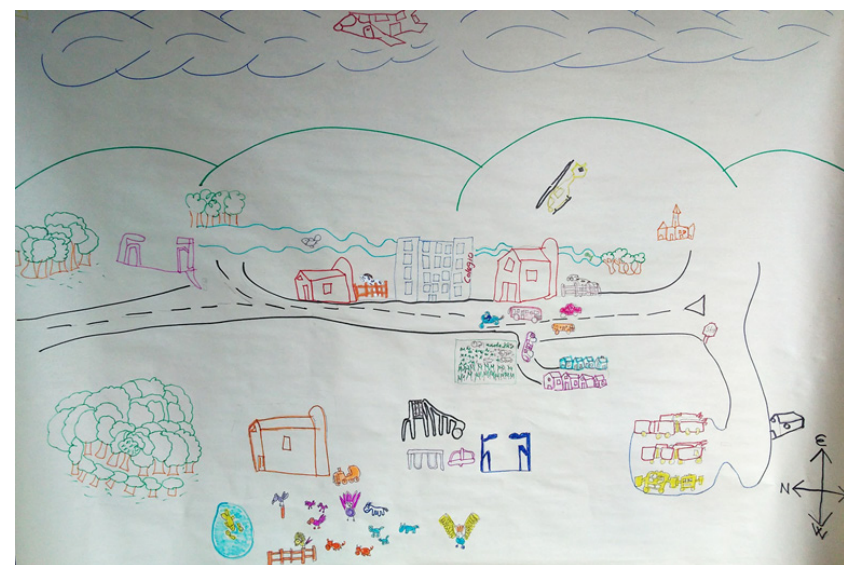

Figura 2. Técnica de mapeo.

Fuente: archivo personal del autor (2016).

La figura 2 hace referencia al tercer mapa realizado por los estudiantes (representando la periferia urbana), en él se recrea la zona colindante a la institución. Esta técnica fue provechosa porque permitió hacer una descripción física del territorio.
Además, sirvió para reconocer los lugares estratégicos y significativos para los estudiantes en los barrios vecinos: Usminia, Villa Anita, Antonio José de Sucre y Usme Centro; siendo estos los parques Villa Alemana y Usminia, la carrera 2 Este y el colegio Diego Montaña Cuellar.

Con el uso de las tres técnicas se logró identificar una serie de semejanzas presentes en los resultados, entre estas: el uso de las zonas verdes como escenarios para jugar e interactuar en grupo, la representación de lo periurbano como un espacio en el que la naturaleza se articula con la ciudad, la presencia de parques y fincas cercanos a la institución, la importancia del colegio como lugar de convivencia, la identificación de zonas naturales asumidas como rurales y el juego como medio de comunicación entre sujeto-territorio.

El juego es una actividad absolutamente indispensable en la formación del niño. Cuando juega está poniendo su imaginación y sus capacidades en práctica. Jugando, el niño conoce el mundo que lo rodea, aprende qué es arriba y qué es abajo, qué es duro y qué es blando. (Jiménez, 2008, p. 179)

De este modo, existe un interés en el uso de la periurbanidad para la diversión de la infancia. También se puede establecer que los juegos al interior del colegio ofrecen vínculos con la naturaleza que aún existe en el sector y que en la institucionalidad de lo escolar tejen lazos de convivencia entre los estudiantes.

\section{Hallazgos y recomendaciones}

Si bien es cierto que la investigación se limitó a unos escenarios específicos en los que hacen presencia los estudiantes, también fue verídico que las instituciones que sirvieron de referente empírico (colegio y ciudad) ofrecieron variables de análisis para interpretar los lazos sociales que construye la infancia en comunidad. Igualmente, los vínculos familiares y barriales de los protagonistas no fueron ajenos a las narrativas que establece la niñez en el territorio, esto último, evidenciado en los resultados que ofrecieron los grupos de discusión.

Como consecuencia de esta investigación se puede decir que los protagonistas construyen lazos 
sociales que se fortalecen en la territorialidad de Usme. En general, las niñas y los niños hacen uso de los espacios que le ofrece la zona periférica de la localidad para desarrollar su subjetividad como seres sociales, la cual está determinada por el juego, el uso de las zonas verdes y las relaciones que establecen entre pares. Se trenza un vínculo entre el contexto territorial y los estudiantes que se puede observar en los juegos que practican, tanto al interior del colegio como fuera de este, manifestaciones que tienen su origen y consolidación en la zona porosa de Usme, la cual se presentó como un escenario periurbano.

También se pudo concluir que la infancia re-significa el vocabulario juvenil dominante en esta zona limítrofe de la ciudad, al otórgale nuevos sentidos que son utilizados en la cotidianidad del colegio, proceso que se observó en el trabajo de campo dentro de la institución. En cuanto a las tradiciones rurales, estas aún existentes en la periferia; los estudiantes las identifican, pero no se apropian de estas para construir saberes dentro del colegio; aunque un determinado número de estudiantes conviven con familiares que trabajan en el campo y en sus expresiones comunicativas, creativas y lúdicas predominan las narrativas urbanas de la localidad.

Se evidencia una tensión entre periurbanidad-infancia que puede ser objeto de análisis desde la institucionalidad de la escuela; esto es observado en el uso que hacen los estudiantes del territorio. Para problematizar dicha tensión, la investigación aporta: el juego como medio de conexión entre sujeto-territorio, el vínculo entre naturaleza-ciudad, la institución como lugar de transito de saberes desde el territorio, el contacto entre lo periurbano y la escuela y la identificación que hacen los estudiantes de la periferia urbana en la que se localiza la institución.

Si se tiene en cuenta el gran número de establecimientos educativos que se emplazan en esta zona limítrofe y la densa población de niñas, niños y adolescentes en edad de escolarización que habitan en la localidad, las zonas periféricas de la ciudad representan una plataforma de acción que se puede problematizar desde el ejercicio educativo. Por lo anterior, podrían aparecer apuestas pedagógicas que exploren el contexto fronterizo desde el análisis de la infancia y, además, pueden surgir líneas de investigación como: pedagogía periurbana; educación y frontera rural-urbana; infancia, territorio y escuela; estrategias de convivencia escolar en la periferia; conservación de lo periurbano desde el escenario educativo; didáctica y espacio público; entre otras, que indaguen sobre lazos que se tejen entre infancia-territorio desde la periferia bogotana.

Sumado a lo anterior, irrumpieron algunos interrogantes que giraron alrededor de la articulación infancia-territorio; primero, sobre la pertinencia de teorizar lo periurbano desde la institucionalidad de la escuela: ¿cuáles son los alcances que tiene el juego en la conexión infancia-territorio?, ¿de qué manera el vínculo escuela-periurbanidad ofrece dinámicas de lectura sobre el territorio?; segundo, sobre la relación periferia urbana-pedagogía: ¿qué tipo de experiencias pedagógicas pueden dinamizar prácticas de convivencia sobre la periferia?, ¿cómo podría el crecimiento de lo urbano encausar ejercicios didácticos que problematicen el contexto geo-cultural de los estudiantes? Son estos algunos de los cuestionamientos que sembró la investigación y que podrían ser desarrollados en otros trabajos pedagógicos que tengan como objeto de estudio el entramado infancia-territorio desde la periferia urbana.

Para ampliar los alcances que puede tener la relación infancia-territorio en la construcción de lazos sociales y en la consolidación de saberes, en posteriores investigaciones se sugiere tener en cuenta categorías o variables de análisis que se limitaron o no fueron abordas en este trabajo, siendo estas: mayor relevancia al contexto familiar y barrial de los estudiantes, antecedentes personales y del núcleo familiar, contexto cultural separado del territorio escolar, medios de comunicación y tiempos más extensos para la intervención de campo.

La intención de este documento es servir de consulta para otras investigaciones pedagógicas que tengan como referente empírico las zonas contiguas de las ciudades, en especial, los bordes urbanos de Bogotá. Además, propone una tensión entre sujeto-territorio que puede ser analizada desde el escenario escolar. Lo anterior, aunado a la necesidad de complejizar sobre la utilidad de la periferia en la configuración de prácticas pedagógicas y ejercicios didácticos en pro de la infancia residente en estos sectores. 
Se sugirió acercar el poder de lo periurbano y sus expresiones a la institución educativa. En este caso, su análisis contribuyó en la elaboración del plan de estudio del área de Ciencias Sociales, debido a que desde la lectura de su contexto propone una suerte de incertidumbres en torno a la interacción entre infancia y territorio que permite empoderar a los sujetos de expresiones territoriales hacía la convivencia. Por último, con estudiantes de la UPZ se planteó el adelanto de un proyecto edu-comunitario sobre la periurbanidad, el cual busca la articulación entre la Casa de la Cultura Fundación Cultural y Artística Antífona, de Comuneros, con el colegio Diego Montaña Cuellar.

\section{Referencias}

Aguilar, A. (2002). Las mega-ciudades y las periferias expandidas. Ampliando el concepto en Ciudad de México. Revista Latinoamericana de Estudios Urbano Regionales, EURE, 28(85), 121-149. https://doi. org/10.4067/S0250-71612002008500007

Alcaldía Mayor de Bogotá D. C. (2004). Decreto 190 de 2004. Recuperado de http://www.alcaldiabogota.gov.co/sisjur/normas/Norma1.jsp?i=13935

Bahamón, G. (2009). Perímetros urbanos. Análisis del proceso de delimitación de espacialidades urbanas, un estudio de caso en Medellín (tesis de maestría). Universidad Nacional de Colombia, Medellín, Colombia. Recuperado de http://www.bdigital.unal.edu. $\underline{\mathrm{co} / 3418 / 1 / 70561800.2009 \text { 1.pdf }}$

Delgado, J. (2003). La urbanización difusa, arquetipo territorial de la ciudad-región. Sociológica, 18(51), 13-48.

Departamento Administrativo Nacional de Estadística (Dane) (2011). Estimado y proyección de población nacional, departamental y municipal total por área 1985-2020. Recuperado de https://www.dane.gov.co/index.php/ estadisticas-por-tema/demografia-y-poblacion/ proyecciones-de-poblacion
Infante, R. y Jiménez, A. (2006). Infancia, ciudad y narrativa. Artes y Ciencias Sociales, 8(1), 268-286.

Jiménez, A. (2007). Infancia y ciudad. Una mirada desde las narrativas populares urbanas en Bogotá. Revista Colombiana de Educación, 53(2), 150-175.

. (2008). Historia de la infancia en Colombia: crianza, juego y socialización, 1968-1984. Anuario Colombiano de Historia Social y de la Cultura, 35(2), 155-188.

Molina, W. (2011). Identidad regional en Magallanes, sus expresiones simbólicas y territoriales. Magallania, 39(1), 59-69. https://doi. org/10.4067/S0718-22442011000100003

Rockwell, E. (1993). Etnografía y teoría de la investigación cualitativa. Recuperado de https://cazembes.files.wordpress.com/2016/05/ elsie-rockwell-etnografc3ada-y-teorc3ada-de-la-investigacic3b3n-educativa6.pdf - (2009). La experiencia etnográfica: historia y cultura en los procesos educativos. Buenos Aires: Paidós.

Sandín, E. y Paz, M. (2003). Investigación cualitativa en educación. Fundamentos y tradiciones. Madrid: Mc Graw-Hill Interamericana.

Secretaria Distrital de Planeación (SDP) (2010). Bogotá. Ciudad de estadísticas. Boletín No. 22. Densidades urbanas el caso Bogotá. Recuperado de http://www.sdp.gov.co/portal/page/ portal/PortalSDP/InformacionTomaDecisiones/ Estadisticas/Bogot\%E1\%20Ciudad\%20de\%20 Estad\%EDsticas/2010-2/DICE105-CartillaDensidadUrbana-2010.pdf

Suárez, Z. (2012). Etnografía crítica. Surgimiento y repercusiones. Revista Comunicación, 21(1), 16-24.

Vargas, I. (2016). ¿Cómo se concibe la etnografía crítica dentro de la investigación cualitativa? Revista Electrónica Educare, 20(2), 1-16. https://doi.org/10.15359/ree.20-2.25

Zuluaga, O. (1999). Pedagogía e historia. La historicidad de la pedagogía. La enseñanza, un objeto de saber. Medellín: Universidad de Antioquia. 